\title{
On the locality of parallel transport of heat carrying electrons in the SOL
}

\author{
A.V. Chankin* and D.P. Coster \\ Max-Planck-Institut für Pasmaphysik, 85748 Garching, Germany
}

\begin{abstract}
A continuum Vlasov-Fokker-Planck code KIPP is used to assess the degree of locality of parallel transport of heat carrying electrons (HCE) in collisional SOLs. It is shown that for typical SOL collisionalities, the HCE are marginally collisional, justifying attempts to parameterize kinetic code results of transport parameters such as parallel heat flux and ionelectron thermoforce in the present 2D fluid codes. A kinetic solution for the case of $90 \%$ recycling at the target and factor $10 \mathrm{~T}_{\mathrm{e}}$ drop along the field line is also presented, showing the degree of heat flux 'limiting' upstream and 'enhancement' downstream, compared to predictions of the Braginskii’s (or Spitzer-Härm's) formulas. Possible causes of these features are discussed.
\end{abstract}

JNM keywords: Plasma-Materials Interaction, Plasma Properties PSI-20 keywords: kinetic, electrons, SOL, transport, collisionality PACS: 52.55.Fa, 52.20.Fs, 52.25.Dg, 52.25.Fi

*Corresponding author address: Max-Planck-Institut für Plasmaphysik, Boltzmannstr. 2, 85748 Garching, Germany

*Corresponding author E-mail: Alex.Chankin@ipp.mpg.de

Presenting author: Dr A.V. Chankin

Presenting author E-mail: Alex.Chankin@ipp.mpg.de 


\section{Introduction}

Kinetic calculations of parallel plasma transport in the scrape-off layer (SOL) and divertor may be very CPU time consuming due to the need to set the time step ( $\Delta \mathrm{t})$ to a fraction of the Coulomb collision time, $\tau_{\mathrm{e}, \text { coll. }}$. The latter drops to very low levels in cold and dense divertor plasmas owing to $\tau_{\mathrm{e}, \text { coll. }} \propto \mathrm{T}_{\mathrm{e}}^{3 / 2} / \mathrm{n}_{\mathrm{e}}$. The requirement to adjust $\Delta \mathrm{t}$ to low $\tau_{\mathrm{e}, \text { coll. }}$ in the divertor, combined with long calculation times necessary to describe the (much slower) evolution of the upstream plasma, may prevent completion of calculations (e.g. reaching a steady state solution) in a reasonable amount of time. There is therefore a strong demand for analytical and computational efforts that would, ideally, allow parameterization of main kinetic results such as the ratio of parallel electron heat conductivity to that calculated according to Braginskii's (or Spitzer-Härm's) formulas, $\chi_{\| \mathrm{e}} / \chi_{\| \mathrm{e}, \mathrm{Brag}}$, against plasma parameters and their spatial derivatives. This however may only be possible if super-thermal electrons carrying the bulk of the parallel heat flux (Heat Carrying Electrons, or HCE) are not collisionless, otherwise the heat transport becomes strongly non-local and only fully kinetic calculations can yield trustable results. Due to its accuracy, KIPP (KInetic Code for Plasma Periphery) [1-3] is an ideal tool to address the issue of a possible parameterization of kinetic code results. Various aspects of parallel transport of HCE, related to their collisionality, are discussed in the paper. Earlier contributions towards inclusion of non-local effects in fluid models for the parallel plasma transport, in particular the use of integral kernels for the calculation of parallel heat conduction, can be found e.g. in Ref. [4]. 


\section{Collision mean free path for a typical/representative heat-carrying electron (HCE)}

KIPP uses dimensionless parameters described in [1]: dimensionless distance along the field line $\tilde{\mathrm{s}}_{\|}=\mathrm{s}_{\|} / \mathrm{s}_{\mathrm{o}}$, where $\mathrm{s}_{\mathrm{o}}=\mathrm{v}_{\mathrm{o}} \tau_{\mathrm{o}}, \mathrm{v}_{\mathrm{o}}=\sqrt{\mathrm{T}_{\mathrm{o}} / \mathrm{m}_{\mathrm{e}}}\left(\mathrm{T}_{\mathrm{o}}-\right.$ some reference electron temperature $)$, collision time $\tau_{\mathrm{o}}=\mathrm{v}_{\mathrm{o}}^{3} \mathrm{~m}_{\mathrm{e}}^{2} /\left(4 \mathrm{e}^{4} \mathrm{n}_{\mathrm{o}} \Lambda_{\mathrm{o}}\right)$. Distance $\mathrm{s}_{\mathrm{o}}$ is calculated upstream, at the stagnation point, where dimensionless temperature and density $\widetilde{T}=\tilde{n}=1$. Hence, the spatial size of the grid in $\tilde{s}_{\|}$corresponds to the number of collision mean free paths (m.f.p.) calculated at the stagnation point, along parallel distance, which is an approximate measure of collisionality of the KIPP case. It has to be noted that, owing to different definitions of collision time, SOL (electron) collisionality $v_{\mathrm{SOL}}^{*}$, defined by Stangeby ([5], p.194), is by factor 3.76 smaller than collisionality defined by using $1 / \tau_{\mathrm{o}}$ here, which may be referred to as $v_{\mathrm{KIPP}}^{*}$. Below, tilde signs $(\sim)$ for dimensionless parameters will often be omitted.

For the study of the HCE collisionality, a strongly collisional KIPP case, as close as possible to assumptions of analytical theory of infinite collisionality, was chosen, with $\tilde{\mathrm{s}} \|=1000$, small $(\approx$ 10\%) $\mathrm{T}_{\mathrm{e}}$ drop from the stagnation point to the target, and fine velocity grid: 400 cells in $\mathrm{v}_{\|}$and 200 in $v_{\perp}$, with the maximum velocity $v_{\max }=7 v_{o}$ (see details of this case in Fig. (1) of [3]). The electron distribution function $f_{e}$ was selected in the middle of the spatial grid, where the heat conduction coefficient $\chi_{\mathrm{e}}$ was found to be very close to the Braginskii's (Eq. (2.12) of [6]). In all KIPP cases referred to here the number of spatial cells is 63 , which corresponds to the number of processes used in the MPI parallelization (64) minus one processor dedicated to some common tasks including output of results. 
Simple estimates of the collisionality of the HCE are based on the scaling $\lambda_{\mathrm{ei}} \propto \mathrm{v}_{\mathrm{e}}^{4}$. From Chodura's calculations [7], the maximum of the parallel electron heat flux density $\mathrm{q}_{\mathrm{e} \|}=\int \mathrm{f}_{\mathrm{e}} \frac{\mathrm{m}_{\mathrm{e}} \mathrm{v}_{\mathrm{e}}^{2}}{2} \mathrm{v}_{\mathrm{e} \|} \mathrm{dv}$ is reached at $\mathrm{v} / \mathrm{v}_{\text {th }}=3.74$, with $\mathrm{v}_{\text {th }}=\sqrt{\mathrm{T}_{\mathrm{e}} / \mathrm{m}_{\mathrm{e}}}$, from where one may conclude that the collisionality of the HCE is by factor $\sim 3.74^{4} \approx 194$ lower than that of thermal electrons, which would make HCE almost collisionless in many plasma conditions encountered in the SOL and divertor. In reality, the collisionality of the HCE is much stronger, when more refined estimates of the m.f.p. are made.

Fig. 1 shows the dependence of the energy density flux on absolute electron velocity and the integral of this quantity over velocity, calculated by KIPP. The maximum of the heat flux density is reached at $\mathrm{v} / \mathrm{v}_{\mathrm{th}}=3.45$, which is lower than in Chodura's calculations but is very close to the value (3.44) obtained with the kinetic code ALLA (Fig. 11 of [8]), where heat flux density was plotted vs. electron energy. The difference with the Chodura's result can be explained by the use of an approximate collision operator in [8]. Chodura concluded that most of the contribution to electron heat flux comes from electrons with energy $\sim 10 \mathrm{~T}_{\mathrm{e}}$. A more precise estimate following from $\mathrm{v} / \mathrm{v}_{\mathrm{th}}=3.45$ gives electron kinetic energy of $3.45^{2} \mathrm{~T}_{\mathrm{e}} / 2=5.95 \mathrm{~T}_{\mathrm{e}}$ for the peak in energy flux. Alternatively, one could also take the velocity corresponding to a half of the integrated energy flux (Fig. 1), v/ $\mathrm{v}_{\text {th }}=3.75$ as a characteristic HCE velocity. This would give $7.03 \mathrm{~T}_{\mathrm{e}}$ for the HCE energy. We will be assuming here that the ratio $v / \mathrm{v}_{\text {th }}$ for the characteristic HCE is somewhere between 3.45 and 3.75 . 
In order to estimate the collision m.f.p. $\lambda_{\text {ei }}$ for HCE even more precisely, one needs to know separately their characteristic $\mathrm{v}_{\|}$and $\mathrm{v}_{\perp}$. Fig. 2 shows the $2 \mathrm{D}$ contour plot of the heat flux density $\mathrm{q}_{\mathrm{e} \|}$ vs. $\mathrm{v}_{\|}$and $\mathrm{v}_{\perp}$ obtained in the same KIPP run as the one used in Fig. 1. The peak in $\mathrm{q}_{\mathrm{e} \|}$ is reached at $\mathrm{v}_{\|}=2.74 \mathrm{v}_{\mathrm{th}}, \mathrm{v}_{\perp}=1.92 \mathrm{v}_{\mathrm{th}}$, corresponding to $5.60 \mathrm{~T}_{\mathrm{e}}$ of the kinetic energy, which is < $5.95 \mathrm{~T}_{\mathrm{e}}$ following from Fig. 1. In order to normalize to the latter value, a 'flux expansion' coefficient 1.0308 of the contour plot Fig. 2, reflecting wider density flux distributions at higher energies (wider separation of the isocontour lines at higher $\mathrm{v}_{\|} / \mathrm{v}_{\text {th }}$ and $\mathrm{v}_{\perp} / \mathrm{v}_{\text {th }}$ ), can be applied to the velocities, leading to slightly corrected values: $\mathrm{v}_{\|}=2.82 \mathrm{v}_{\mathrm{th}}, \mathrm{v}_{\perp}=1.98 \mathrm{v}_{\mathrm{th}}$. Hence, 2.82 can be taken as a factor of an increase in the HCE parallel velocity compared to the thermal velocity $\left(\mathrm{v}_{\| \mathrm{hce}} / \sqrt{\mathrm{T}_{\mathrm{e}} / \mathrm{m}}\right)$. An increase in the collision time is approximately given by $\frac{\tau_{\mathrm{hce}}}{\tau_{\mathrm{th}}} \approx\left(\frac{\varepsilon_{\mathrm{hce}}}{1.5 \mathrm{~T}_{\mathrm{e}}}\right)^{3 / 2}$, where $\varepsilon=5.95 \mathrm{~T}_{\mathrm{e}}$. Altogether, a factor of the m.f.p. increase of the HCE compared to thermal electrons is given by $\frac{\lambda_{\text {hce }}}{\lambda_{\text {th }}} \approx\left(\frac{\varepsilon_{\text {hce }}}{1.5 \mathrm{~T}_{\mathrm{e}}}\right)^{3 / 2} \times \frac{\mathrm{v}_{\| \mathrm{hce}}}{\sqrt{\mathrm{T}_{\mathrm{e}} / \mathrm{m}}}=22.3$. If one used the characteristic HCE energy of $7.03 \mathrm{~T}_{\mathrm{e}}$ instead of $5.95 \mathrm{~T}_{\mathrm{e}}$, one would have obtained: $\lambda_{\text {hce }} / \lambda_{\text {th }}=31.1$. One may therefore conclude that the HCE collisionality is approximately by factor 20 to 30 lower than that of thermal electrons. For cases with lower collisionalities and steeper $\mathrm{T}_{\mathrm{e}}$ profiles, maxima in $\mathrm{q}_{\mathrm{e} \|}$ are achieved at somewhat larger $\mathrm{v} / \mathrm{v}_{\mathrm{th}}$. Still, this doesn't change the conclusion that for typical SOLs with dimensionless electron collisionality for thermal electrons in the range of $20-$ 50 [5] HCE appear to be marginally collisional. One may therefore attempt to parametrise macroscopic quantities such as electron parallel heat conduction against plasma parameters and their $1^{\text {st }}$ and $2^{\text {nd }}$ derivatives. 


\section{KIPP case with the large $T_{e}$ drop}

Fig. 3 shows a quasi steady state solution of the KIPP case with $\sim 10$ times $\mathrm{T}_{\mathrm{e}}$ drop from the stagnation point to the Debye sheath (target) achieved by an artificial exponential power sink profile near the target with the spatial decay length of $1 / 8^{\text {th }}$ of the whole domain, with the feedback on the $\mathrm{T}_{\mathrm{e}}$ in the last cell bordering the target, aiming at maintaining it at 0.1 (in relative units). In addition, power and particle sources with the same profile were specified near the stagnation point, feedbacked on the stagnation point parameters to maintain $\widetilde{\mathrm{T}}_{\mathrm{e}}=\tilde{\mathrm{n}}_{\mathrm{e}}=1$, and the particle source near the target with the same profile was used, adjusted to match the particle sink calculated by assuming $90 \%$ ion recycling at the target. The spatial grid was $100 \mathrm{~s}_{\mathrm{o}}$ wide, with $\mathrm{s}_{\mathrm{o}}$ calculated for parameters at the stagnation point, hence, corresponding to 100 electron collision m.f.p. at these parameters, or to $v_{\mathrm{SOL}}^{*} \approx 27$ upstream, using Stangeby's collisionality. In order to cover wide range of $\mathrm{T}_{\mathrm{e}}$ and $\nabla_{\|} \mathrm{T}_{\mathrm{e}}$ variations, slightly exponential velocity and spatial grids, with every subsequent grid size larger than the previous by factor 1.03 for both grids, were used. All other assumptions were most generic, with the ionization effect achieved by simply scaling the density of the electron distribution function $f_{e}$ and an automatic adjustment of $T_{i}$ to match $T_{e}$, hence, implying unspecified ion heat sources. A simple fluid ion model (for deuterium) without parallel viscosity was used. The ion Mach number at the target reached 1.87 (the minimum Mach number was set to 1 , but the maximum was not limited). In order to obtain correct transport coefficients, a very small time step $\Delta \tilde{\mathrm{t}}=0.00155$ was chosen, which would translate into the time step $0.1 \tau_{\mathrm{o}}$ (using the local $\tau_{\mathrm{o}}$ ) for the much more collisional plasma in the cell adjacent to the target. 
As one can see from Fig. 3, the ratio of the parallel electron heat conduction $\mathrm{q}_{\| \mathrm{e}}$ to that calculated according to the Braginskii's formula, or, which is the same, the ratio of parallel heat conductivities, $\chi_{\| \mathrm{e}} \mathrm{\chi}_{\| \mathrm{e}, \mathrm{Brag}}$, is $<1$ ('heat flux limiting') upstream and $>1$ downstream ('heat flux enhancement'), close to the target, in accordance with many earlier kinetic results (see e.g. review paper [9]). Especially pronounced is a rather wide zone of the 'flux limiting' far away from the target. Similarly, in the same region the ratio of the parallel electric field caused by the e-i thermoforce, over the $\mathrm{T}_{\mathrm{e}}$ gradient, $-\mathrm{eE}_{\|, \text {therm }} / \nabla_{\|} \mathrm{T}_{\mathrm{e}}$, is substantially below the theoretical level for strongly collisional plasmas, 0.71 .

For parameterization of KIPP results, various dimensionless parameters, including local plasma collisionality, and $1^{\text {st }}$ and $2^{\text {nd }}$ derivatives of $T_{e}$ and $n_{e}$, will be tried in the future. Also, different KIPP runs, obtained by varying the recycling level, and using the ion fluid model of SOLPS (B2), as well as its fluid neutral model, are planned. One of the most important dimensionless parameters is likely to be plasma collisionality calculated by taking the parallel $\mathrm{T}_{\mathrm{e}}$ decay length, $\lambda_{\mathrm{T}_{\mathrm{e}}}=-\mathrm{T}_{\mathrm{e}} / \nabla_{\|} \mathrm{T}_{\mathrm{e}}$, as a characteristic distance: $v_{\lambda_{\mathrm{T}}}^{*} \propto \lambda_{\mathrm{T}_{\mathrm{e}}} / \mathrm{v}_{\mathrm{Te}} \tau_{\mathrm{o}}$. It is plotted in Fig. 3 (bottom box). Values 300 imply that HCE for this case are marginally collisional, as one has to bear in mind the factor 20-30 reduction in the HCE collisionality compared to that of thermal electrons and the factor 3.76 reduction in their collisionality compared to the standard, Stangeby's, definition of dimensionless collisionality. Since $\chi_{\| \mathrm{e}} / \chi_{\| \mathrm{e}, \mathrm{Brag}}$ is seen rising for $\mathrm{s} \|$ from 20 to 80 , while $v_{\lambda_{T_{e}}}^{*}$ remains nearly constant, it is clear that $v_{\lambda_{T_{e}}}^{*}$ along would be insufficient to parameterize the results, and the second derivative of $\mathrm{T}_{\mathrm{e}}$ will probably be required, as well as, possibly, density derivatives. 


\section{Influence of the stagnation point and Debye sheath}

In KIPP, presently the left-right symmetry of $f_{e}$ at the stagnation point is assumed: $\mathrm{f}_{\mathrm{e}}\left(-\mathrm{v}_{\|}\right)=\mathrm{f}_{\mathrm{e}}\left(+\mathrm{v}_{\|}\right)$. This certainly makes an impact on the $\chi_{\| \mathrm{e}} / \chi_{\| \mathrm{e}, \mathrm{Brag}}$ ratio, since right at this point no heat flux is possible. Despite the rise in $v_{\lambda_{\mathrm{e}}}^{*}$ towards the stagnation point, which should make the plasma more collisional and raise the $\chi_{\| \mathrm{e}} / \chi_{\| \mathrm{e}, \mathrm{Brag}}$ ratio, it is seen falling. This indicates that a separate parameterization may be required in the vicinity of the $\mathrm{T}_{\mathrm{e}}$ maximum.

Near the target, the increase in $\chi_{\| \mathrm{e}} / \chi_{\| \mathrm{e}, \mathrm{Brag}}$ may be related to the steepening of the $\mathrm{T}_{\mathrm{e}}$ profile. Oscillations in $\mathrm{q}_{\| \mathrm{e}} / \mathrm{q}_{\| \mathrm{e}, \mathrm{Brag}}$ and $-\mathrm{eE} \mathrm{E}_{\|, \text {therm }} / \nabla_{\|} \mathrm{T}_{\mathrm{e}}$ profiles in the last 3 cells adjacent to the target are not due to the effect of the Debye sheath, but rather due to numerical problems: for the parallel free-streaming scheme adopted, information on the $f_{e}$ at each cell face requires knowledge of $f_{e}$ 's at 4 cells closest to it ( 2 from each side). This is impossible to specify for the last real cell face near the target, and a simpler scheme was used for this cell face.

At the same time, the influence of the Debye sheath itself on the rise in the $\chi_{\| \mathrm{e}} / \chi_{\| \mathrm{e}, \mathrm{Brag}}$ ratio at the target is probably very limited. It is clear that the truncation of $\mathrm{f}_{\mathrm{e}}\left(\mathrm{v}_{\|}\right)$at the Debye sheath for high negative velocities (electrons beyond certain parallel energy impacting on the target get deposited to it, hence, no such electrons are reflected back to the plasma) automatically creates parallel electron power flux to the target. However, this effect should be small for cases with large $T_{e}$ drops from the stagnation point to the target. A dedicated KIPP case with the plasma flowing to the target at the ion sound speed with spatially constant $n_{e}$ and $T_{e}$ (with power 
supplied into boundary cells in order to maintain spatially constant $\mathrm{T}_{\mathrm{e}}$ ) was run in order to assess how long-distant is the effect of the Debye sheath on the target heat flux created by it. The maximum in $\mathrm{q}_{\| \mathrm{e}}$, reached at the target, was found to decay by factor 10 over a distance of $11.8 \mathrm{~s}_{\mathrm{o}}$ (11.8 collisional m.f.p.). For the case shown in Fig. 3, taking into account a much higher plasma collisionality near the target, this would translate into the distance $\Delta \mathrm{s}_{\|}=0.51$, which is close to the boundary cell size. Hence, the effect of the Debye sheath should decay very quickly.

\section{Summary}

For typical plasma collisionalities encountered in the SOL heat carrying electrons (HCE) are in the marginal collisionality regime. This makes the problem of avoiding very CPU time consuming kinetic calculations for purposes of correctly calculating parallel heat fluxes and their replacement with parameterization formulas particularly challenging. HCE are neither enough collisional, which would have justified simple corrections to fluid results using only plasma parameters and their derivatives, nor are they enough collisionless, which would have enabled one to consider Coulomb collisions as a correction to collisionless solutions. The marginal collisionality however does not rule out devising more sophisticated parameterization formulas for deviations of transport coefficients from results of analytic theory for strongly collisional plasmas.

At present, KIPP results, obtained under very simplified assumptions about plasma density evolution, can only be indicative of the need to develop rather sophisticated parameterization formulas. As a next step, coupling of the electron kinetic description of KIPP with the ion fluid model of SOLPS (B2) and its fluid neutral model is planned. This will provide more material for 
the development of parameterization formulas. The sensitivity of the code results to peculiarities of electron-neutral (and in the future, electron-impurity) interaction mechanisms will also be assessed. It is hoped that, owing to the long distant nature of Coulomb collisions, the influence of electrons' interaction with neutrals and impurities, apart from creating particle and power sources, will be secondary, due to much smaller cross-sections.

\section{Acknowledgement}

This project has received funding from the Euratom research and training programme 20142018.

\section{References}

[1] A.V. Chankin, D.P. Coster, and G. Meisl, Contrib. Plasma Phys. 52 (2012) 500.

[2] G. Meisl, A.V. Chankin, and D.P. Coster, J. Nucl. Mater. 428 (2013) S342.

[3] A.V. Chankin and D.P. Coster, Benchmarks of KIPP: Vlasov-Fokker-Planck Code for Parallel Plasma Transport in the SOL and Divertor, accepted for publication in Contrib. Plasma Physics.

[4] J.T. Omotani and B.D. Dudson, Plasma Phys. Control. Fusion 55 (2013) 055009.

[5] P.C. Stangeby, in The Boundary of Magnetic Fusion Devices, IOP Publishing, Bristol (2000).

[6] S.I.Braginskii, in Transport processes in a plasma, Review of Plasma Physics Vol. 1, A.M.Leontovich (ed.), Consultants Bureau, New York (1965).

[7] R. Chodura, Contrib. Plasma Phys. 32 (1992) 3/4, 219.

[8] O. V. Batishchev, S. I. Krasheninnikov, P. J. Catto et al., Phys. Plasmas 4 (1997) 1672.

[9] W. Fundamenski, Plasma Phys. Control. Fusion 47 R163 (2005). 


\section{Figure captions}

Fig. 1. Parallel electron energy density flux vs. normalized absolute electron velocity (solid line) and its integral (dotted line) obtained in a strongly collisional KIPP case.

Fig. 2. 2D contour plot of the parallel electron energy density flux against normalized parallel and perpendicular electron velocities for the same case as shown in Fig. 1.

Fig. 3. Output profiles for the KIPP case with the factor $10 \mathrm{~T}_{\mathrm{e}}$ drop from the stagnation point to the target: normalized $T_{e}$ (top box), normalized $n_{e}$ ( $2^{\text {nd }}$ from top), ratio of parallel electron heat flux to that calculated according to the Braginskii's formula ( $3^{\text {rd }}$ from top), ratio of electron-ion thermoforce to $n_{e} \times$ parallel $T_{e}$ gradient ( $4^{\text {th }}$ from top), and electron collisionality calculated by taking the parallel $\mathrm{T}_{\mathrm{e}}$ decay length as a characteristic spatial scale (bottom box). 

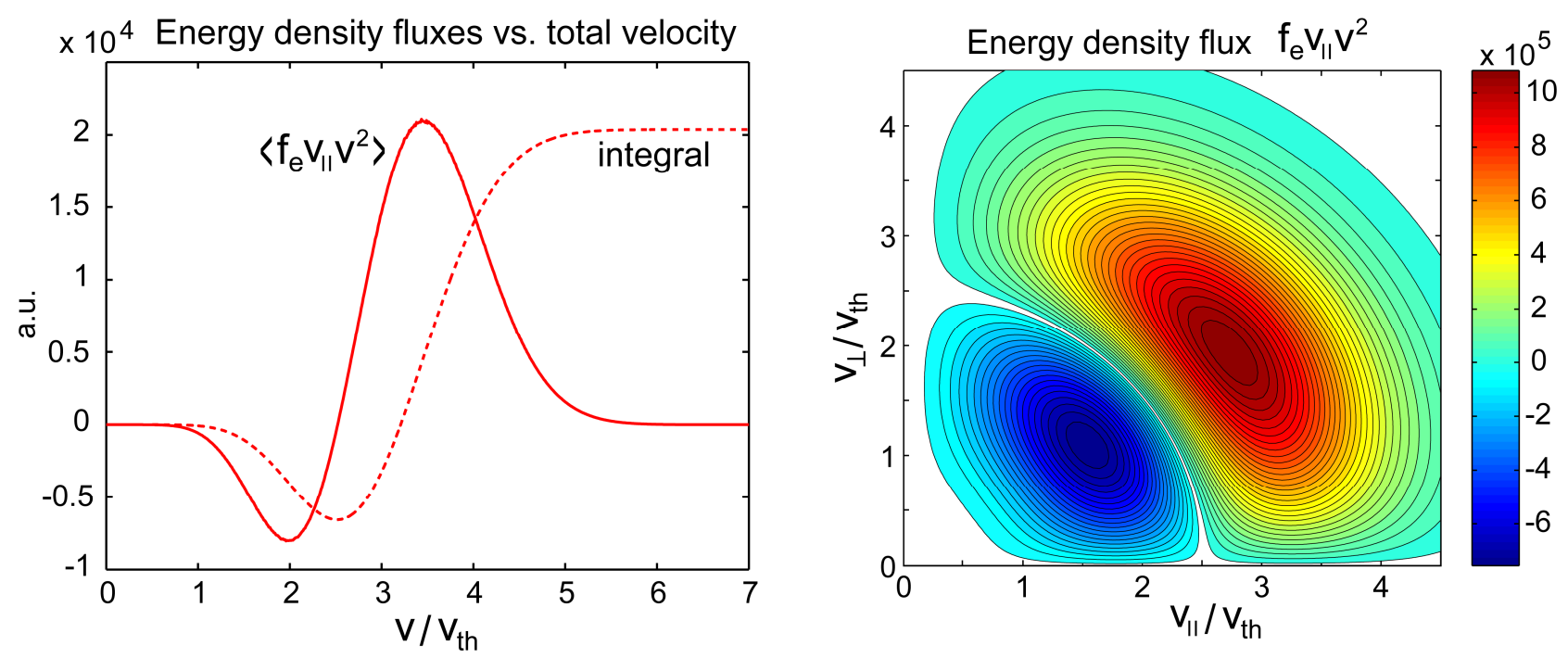

Fig. 1

Fig. 2

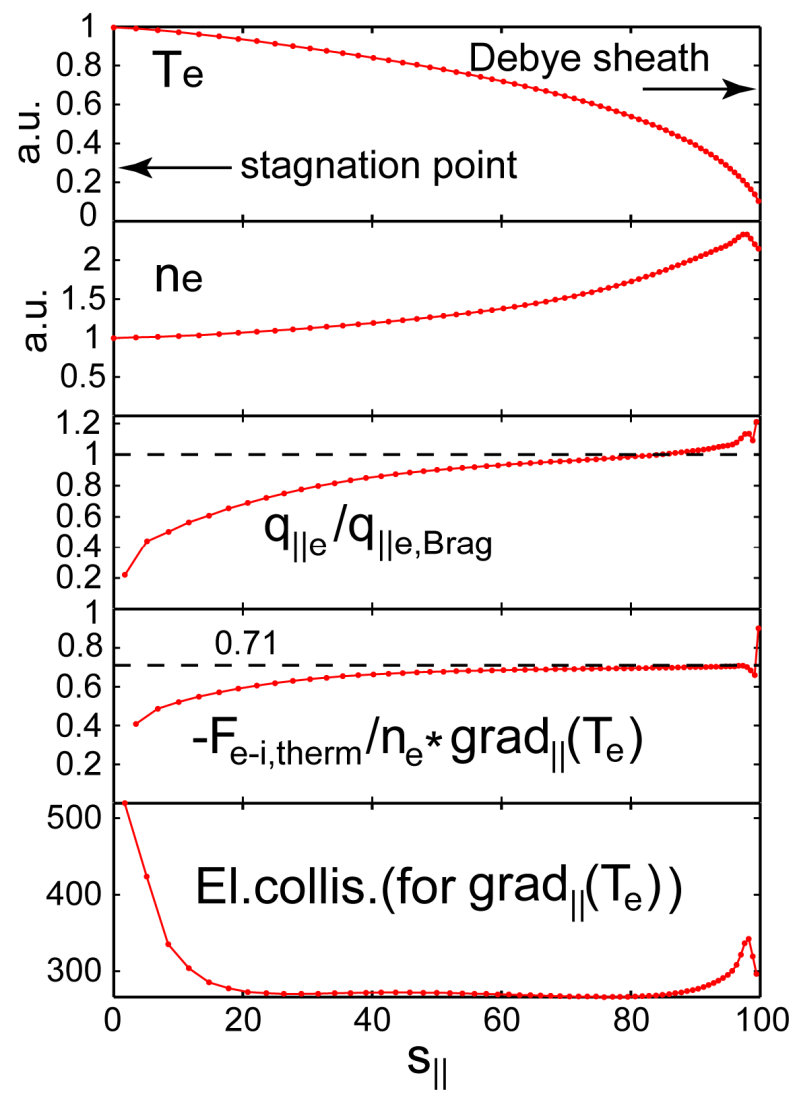

Fig. 3 